\title{
Can we improve pain and sleep in elderly individuals with transcranial direct current stimulation? - Results from a randomized controlled pilot study
}

\author{
This article was published in the following Dove Press journal: \\ Clinical Interventions in Aging \\ 6 June 2017 \\ Number of times this article has been viewed
}

\author{
Marie-Philippe Harvey ${ }^{1,2}$ \\ Dominique Lorrain ${ }^{1,3}$ \\ Marylie Martel ${ }^{1,2}$ \\ Kayla Bergeron-Vezina' \\ Francis Houde ${ }^{1,2}$ \\ Mario Séguin 2,4 \\ Guillaume Léonard 1,2,5 \\ 'Research Centre on Aging, CIUSSS \\ de l'Estrie-CHUS, ${ }^{2}$ Faculty of Medicine \\ and Health Sciences, Université \\ de Sherbrooke, ${ }^{3}$ Department of \\ Psychology, Université de Sherbrooke \\ ${ }^{4}$ Department of Neurosurgery, \\ Centre Hospitalier Universitaire de \\ Sherbrooke, ${ }^{5}$ School of Rehabilitation, \\ Université de Sherbrooke, \\ Sherbrooke, QC, Canada
}

Background: The prevalence of chronic pain and sleep disturbances substantially increases with age. Pharmacotherapy remains the primary treatment option for these health issues. However, side effects and drug interactions are difficult to control in elderly individuals.

Aims: The objective of this study was to assess the feasibility of conducting a randomized sham-controlled trial and to collect preliminary data on the efficacy of transcranial direct current stimulation (tDCS) to reduce pain and improve sleep in older adults suffering from chronic pain.

Methods: Fourteen elderly individuals (mean age $71 \pm 7$ years) suffering from chronic pain and sleep complaints were randomized to receive either anodal tDCS, applied over the primary motor cortex ( $2 \mathrm{~mA}, 20$ minutes), or sham tDCS, for 5 consecutive days. Pain was measured with visual analog scales, pain logbooks and questionnaires, while sleep was assessed with actigraphy, sleep diaries and questionnaires.

Results: There were no missing data for pain and sleep measures, except for actigraphy, that generated several missing data. Blinding was maintained throughout the study, for both the evaluator and participants. Active but not sham tDCS significantly reduced pain $(P<0.05)$. No change was observed in sleep parameters, in both the active and sham tDCS groups (all $P \geq 0.18$ ).

Conclusion: The present study provides guidelines for the implementation of future tDCS studies in larger populations of elderly individuals. M1 anodal tDCS in this population appears to be effective to reduce pain, but not to improve sleep.

Keywords: transcranial direct current stimulation, tDCS, pain, sleep, elderly, actigraphy, aging

\section{Introduction}

Chronic pain and sleep disorders are recognized worldwide as health problems having a significant impact on quality of life and productivity. ${ }^{1,2}$ A vicious circle seems to govern the interaction between pain and sleep disorders, with chronic pain leading to sleep disturbances and poor sleep leading to enhanced pain perception. ${ }^{3-8}$ It is estimated that over $67 \%$ of people experiencing chronic pain also complain about their sleep., 9

The prevalence of chronic pain and sleep disorders increases considerably with age. ${ }^{11-14}$ Pharmacotherapy remains the first line of treatment to alleviate sleep disorders and chronic pain symptoms. ${ }^{14-17}$ Despite the availability of many pharmacological approaches, sleep problems and pain often persist. ${ }^{18}$ In addition, polypharmacy is an important challenge in elderly individuals, a population in which drug interactions are frequent, and often complex to manage. ${ }^{19}$
Correspondence: Guillaume Léonard Research Centre on Aging, CIUSSS de l'Estrie - CHUS, 1036 rue Belvédère Sud, Sherbrooke, QC JIH4C4, Canada

Tel +I 8197802220 Ext 45246

Fax +I 8198206864

Email guillaume.leonard2@

usherbrooke.ca 
Past studies have shown that noninvasive brain stimulation techniques, such as transcranial direct current stimulation (tDCS), can improve sleep, ${ }^{20}$ alleviate pain ${ }^{20-24}$ and reduce medication consumption and related side effects. ${ }^{25,26}$ However, the majority of these studies were performed on young adults suffering from neurogenic pain syndromes, and it is still unknown if tDCS could be a valid treatment option for elderly individuals suffering from chronic musculoskeletal pain. Investigating the effect of tDCS on older populations is crucial given the age-related changes observed in brain function and anatomy. ${ }^{27,28}$ Compared to young adults, elderly individuals show reduced gray matter perfusion and cerebral oxygen metabolic rate. ${ }^{27}$ Reduction in brain volume has also been documented with aging. ${ }^{29}$ Reduction in brain volume increases skull-cortex distance, which can presumably affect the effect of tDCS. ${ }^{29,30}$ The aim of the present study was to assess the feasibility of conducting a randomized, double-blind, sham-controlled trial and to collect preliminary data on the efficacy of tDCS to reduce pain and improve sleep in older adults suffering from chronic musculoskeletal pain.

\section{Materials and methods}

\section{Participants}

Fourteen elderly individuals were included in the study. Individuals were regarded as suitable to participate if they fulfilled the following criteria: 1) aged 60 years or over, 2) reported stable musculoskeletal pain in the previous 3 months or more and 3 ) had a score higher than 7/28 at the Insomnia Severity Index. ${ }^{31}$ Participants with tDCS contraindications, such as neurological or neuropsychiatric conditions (eg, stroke, traumatic brain injury), history of brain surgery or tumor, metallic implants, epilepsy or history of substance abuse or dependence, were excluded. Participants were asked to keep their medication and life habits stable for the duration of the study. Participants were also asked not to consume nicotine and caffeine at least 6 hours before each visit. The experiment took place at the Research Centre on Aging of CIUSSS de 1'Estrie-CHUS (Sherbrooke, QC, Canada). Subjects were all French-speaking community-dwelling individuals. The study was approved by the Research Ethics Committee of CIUSSS de l'Estrie-CHUS, and written informed consent was obtained from all participants. All procedures performed were in accordance with the ethical standards of the local institutional research committee and with the Declaration of Helsinki of 1964 and its later amendments or comparable ethical standards.

\section{Experimental design}

A randomized, parallel, double-blind, sham-controlled design was used. The study lasted 19 days and was divided into 3 phases: 1) a 7-day baseline evaluation; 2) a 1-week doubleblind treatment period, which consisted of 5 consecutive daily treatment sessions of sham or active tDCS and 3) a 7-day follow-up period (Figure 1). Throughout the 19 days of the study, daily measures of sleep and pain were recorded. Randomization to sham or active tDCS was performed using a random numbers table with a ratio of $1: 1$, based on order of entry of the participants in the study. The randomized table was designed to assign a total of 16 subjects to the study (8 in each group).

\section{Pain measurements}

Pain intensity was evaluated with a visual analog scale (VAS) and a pain logbook. The VAS is a self-assessment

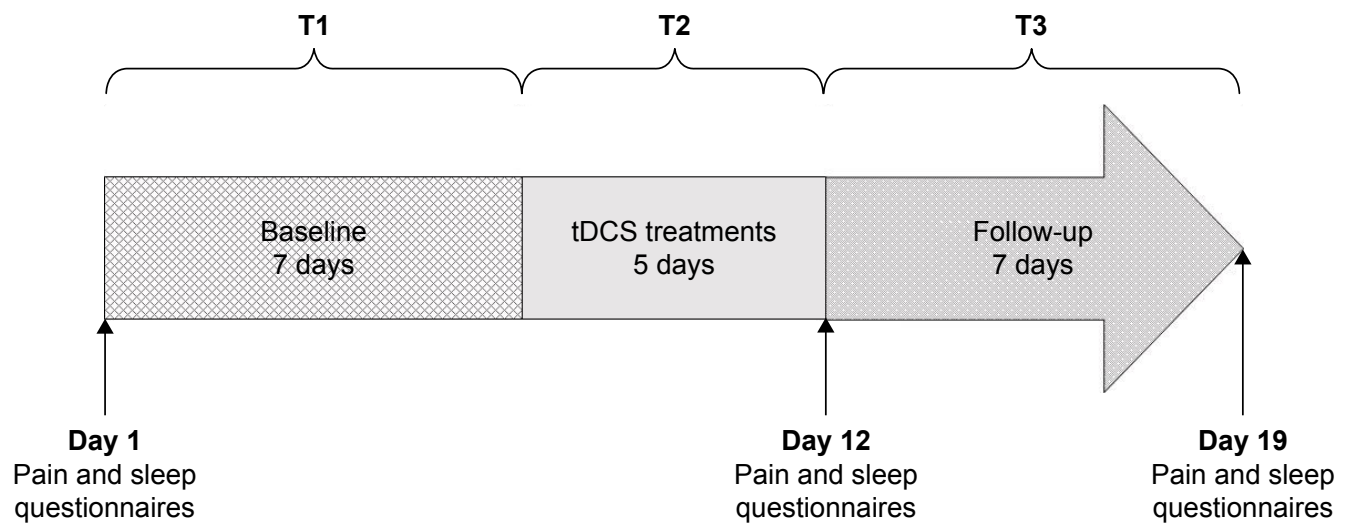

Figure I The study lasted 19 days and was divided into 3 phases: TI (baseline), T2 (tDCS treatments) and T3 (follow-up). Pain and sleep questionnaires were completed at the beginning of the study (day I), after the 5 tDCS sessions (day 12) and after the 7 days of follow-up (day 19). Actigraphic measures were taken from day I to day 19. Pain and sleep logbooks were completed each day, at home, by the participants, from day I to day 19 .

Abbreviation: tDCS, transcranial direct current stimulation. 
scale of $10 \mathrm{~cm}$ that ranges from "no pain" $(0 \mathrm{~cm})$ to "the worst imaginable pain" $(10 \mathrm{~cm})$. This scale is widely used to evaluate pain outcome in studies, and its validity is well established. ${ }^{32,33}$ Participants had to rate pain intensity in the laboratory with a VAS once on day 1 and day 19 , and before and after each tDCS session (day 8 to day 12). In addition to the VAS pain score, participants were asked to rate their daily pain in a pain assessment logbook containing 3 numerical rating scales (NRSs) ranging from 0 to 10 ( $0=$ no pain; $10=$ maximal pain). These 3 NRSs were used to evaluate the pain felt by the participant 1) at its least during the last 24 hours (minimal pain), 2) at its worst during the last 24 hours (maximal pain) and 3) on average in the last 24 hours (average pain). The pain assessment logbook was filled out by the participant at the end of each day throughout the duration of the study. The NRSs have been shown to be reliable and valid to measure pain intensity in elderly patients with persistent pain. ${ }^{34}$ Pain assessments with the VAS were used in the laboratory to measure current pain, while the pain logbook was used to reflect the pain for each day. Although the pain intensity measurement is essential, it captures only part of the pain experience in older patients and should be supplemented by other pain measures. ${ }^{33,34}$ Consequently, pain questionnaires such as the McGill Pain Questionnaire (MPQ) and the Short Form of the Brief Pain Inventory (SF-BPI) were used to assess qualitative aspects of pain and physical functioning, respectively. These questionnaires were completed 3 times during the study (ie, on day 1, 12 and 19; Figure 1). The validity and reliability of both questionnaires have been previously documented. ${ }^{35-38}$

\section{Sleep measurements}

Sleep efficiency, which is the ratio of total time spent in bed to total time actually spent sleeping, was evaluated with actigraphy (Model Actiwatch; Philips - Respironics, Murrysville, PA, USA). Actigraphic records were also used to quantify nocturnal awakenings and estimate sleep-onset latency. Participants were asked to wear the actigraph device on their nondominant wrist throughout the duration of the study. Actigraphy has been shown to be a reliable method of recording activity during sleep. ${ }^{39,40}$ Actigraphic recordings were supplemented with the data collected in the sleep diary; during their participation in the study, from day 1 to day 19, participants were asked to fill out a sleep diary collecting information on the quality and quantity of sleep that they perceived. In addition to the actigraph and the sleep diary, the Pittsburgh Sleep Quality Index (PSQI) was used to assess the participant's sleep quality. The PSQI showed a strong validity and reliability in both clinical and nonclinical samples. ${ }^{41}$ Finally, 1 question of the SF-BPI, based on a 0-10 NRS, and labeled "Circle the one number that describes how, during the past 24 hours, pain has interfered with your sleep", was used to assess pain interference with sleep $(0=$ does not interfere; $10=$ completely interferes).

\section{tDCS protocol}

Participants were seated comfortably in an armchair during the $5 \mathrm{tDCS}$ treatment sessions. The stimulations for a given subject were always done by the same investigator who was different from the evaluator. The investigator was responsible for the assignment of participants to the active or sham tDCS group, while the evaluator and the participants were blinded. The stimulations were always given in the afternoon or in the evening to get as close as possible to the actual time period of the night of sleep. Direct current was transferred to the subject by a saline-soaked pair of surface sponge electrodes $(5 \times 7 \mathrm{~cm})$ and delivered by a constant current stimulator, battery-driven, $1 \times 1$ tDCS device (Model 1300-A; Soterix Medical Inc, New York, NY, USA). Participants received either anodal stimulation of the primary motor cortex (M1) or sham stimulation of M1. The anodal electrode was placed over M1, contralateral to the most painful site (C3 or C4 per the electroencephalogram 10/20 system), and the cathodal electrode was placed on the supraorbital area contralateral to the anode. During active tDCS, a constant anodal current of $2 \mathrm{~mA}$ was delivered for 20 minutes. This anodal tDCS procedure is known to increase cortical excitability and to reduce pain. ${ }^{20,21,23,42,43}$ This procedure has also been shown to improve sleep in patients suffering from fibromyalgia. ${ }^{20}$ For the sham stimulation, the electrodes were placed in the same montage as the active tDCS; however, current was applied only for the initial and final 30 seconds. Therefore, the patients felt the ramp-up and ramp-down itching sensation of the current, but received no current for the rest of the stimulation period. This placebo procedure has been validated and is now successfully used in most studies using tDCS. ${ }^{44-46}$ The tDCS device was set by the manufacturer to automatically provide this type of sham stimulation.

\section{Data analysis}

The feasibility of the study was evaluated with regard to the challenges incurred by the data collection and the number of missing data for every pain and sleep measurement tool. Also documented were the number of participants who completed the study, the ability to maintain blinding for the evaluator and participants, as well as randomization issues. 
Preliminary analysis on the effectiveness of the tDCS intervention was also conducted. To facilitate interpretation and to reduce the number of statistical comparisons, pain intensity ratings (pain logbook) and actigraphy measurements (sleep efficiency, sleep-onset latency and number of nocturnal awakenings) were averaged into 3 scores, reflecting the 3 phases of the study (ie, before, during and after tDCS treatment). As shown in Figure 1, T1 represents the 7 days of baseline, T2 corresponds to the 5 days of tDCS treatments and $\mathrm{T} 3$ represents the 7 days of follow-up. The mean values were used for all analyses. Percentages of change were also calculated to directly compare the efficacy of active tDCS and sham tDCS on pain and sleep, based on the following formula: percentage of change $=[$ (score during or after treatment (T2 or T3) - score before treatment (T1))/score before treatment $(\mathrm{T} 1)] \times 100$.

The study was designed to detect a clinically important difference of 2 points on the $0-10$ pain intensity scale. ${ }^{47}$ To detect this difference with $80 \%$ power and a $5 \%$ significance level, we determined that 16 individuals had to be enrolled in the study (estimated standard deviation of 1.3, based on previous tDCS studies ${ }^{23,24}$ ). Because of the low number of subjects, and since visual inspection of the histograms did not allow us to assume that the data were normally distributed, nonparametric tests were used for all the statistical analyses. Specifically, Mann-Whitney tests (continuous variables) and Fisher's exact tests (categorical variables) were used to compare the 2 groups (betweensubject analyses). This allowed us to evaluate if the outcome measures were different between the active and sham tDCS groups. Friedman tests and Wilcoxon signed-rank tests were also used to compare if the intervention affected the outcome measures in each group (intragroup analyses). All tests were performed using SPSS (version 17.0 for Windows; SPSS Inc., Chicago, IL, USA), and differences were considered to be significant if $P<0.05$ was obtained. Bonferroni corrections were applied to prevent type I errors.

\section{Results}

\section{Participants' characteristics}

Sixteen elderly individuals aged between 62 and 84 years (mean age $71 \pm 7$ years; 3 men) were included in the study. Two participants from the active tDCS group dropped out of the study, 1 because of a family event (death of a loved one) and 1 because of a personal matter (withdrawal of driver's license). The demographic and general clinical characteristics of the remaining 14 participants are summarized in Table 1. Participants had no change in medication during the last
3 months. Exploratory analyses and visual inspection of the data revealed that the medication had no effect on the response to tDCS, for pain as well as for sleep.

\section{Pain outcomes}

There were no missing data for all pain outcomes. The participants correctly filled all pain measures and questionnaires. However, the VAS often needed explanations before the participants could fully grasp the concept evaluated. After the first laboratory session, the concept of pain measures was well understood by the participants, and all the daily pain logbooks were completed correctly.

The VAS pain scores obtained on day 1 and day 19, and before and after each tDCS session (day 8 to day 12) in the laboratory are presented in Figure 2. As it can be seen from this figure, no significant change was observed immediately after each tDCS session, for both the active and sham tDCS group. The absence of short-term effect was confirmed by the Friedman and Wilcoxon signed-rank tests, which revealed no significant intragroup differences for both treatment groups (all $P \geq 0.17$ ). Mann-Whitney tests also showed that there were no between-group differences for all time measures (all $P \geq 0.11$ ) suggesting, again, that tDCS has no short-term effect on pain.

Daily average pain ratings, obtained via the pain logbook for the 3 phases of the study, are presented in Figure 3 and in Table 2. As can be seen from this figure, daily average pain ratings decreased among the active tDCS group and slightly increased among the sham tDCS group. This pattern of results was confirmed by the Friedman tests, which revealed a change in pain for both tDCS conditions (both $P \leq 0.03$ ). For the active tDCS group, post hoc Wilcoxon signed-rank tests revealed that there was a significant reduction in daily average pain during (T2; $P=0.04)$ tDCS treatments, when compared to baseline (T1). A significant trend was also observed for T3 $(P=0.06)$. In contrast, post hoc Wilcoxon signed-rank tests showed that sham tDCS slightly increased pain at T3 when compared to T1 $(+0.34 \pm 0.29 ; P=0.04)$.

To better evaluate the effect of active and sham tDCS, percentages of hypoalgesia were calculated (Figure 4). On one hand, active tDCS produced a reduction in pain of $28 \%$ at $\mathrm{T} 2$ and of $49 \%$ at $\mathrm{T} 3$. Both these percentages of pain reduction are clinically significant. ${ }^{48}$ On the other hand, sham tDCS reduced pain by $7 \%$ at T2 and increased pain by $19 \%$ at T3. These percentages of change observed among the sham tDCS group are not clinically significant. Mann-Whitney tests comparing the active tDCS group and the sham tDCS group revealed a significant difference between the groups at T3 $(P=0.008)$, but not at T2 $(P=0.31)$. 
Table I Clinical and demographic characteristics of the participants

\begin{tabular}{|c|c|c|c|c|}
\hline Variable & All participants & Active tDCS & Sham tDCS & $P$-value* \\
\hline Number (n) & 14 & 6 & 8 & \\
\hline Gender $(\mathrm{F} / \mathrm{M})^{\ddagger}$ & $11 / 3$ & $5 / 1$ & $6 / 2$ & 1.00 \\
\hline Hand dominance (right/left) $)^{\ddagger}$ & $14 / 0$ & $6 / 0$ & $8 / 0$ & 1.00 \\
\hline \multicolumn{5}{|l|}{ Age (years) } \\
\hline Mean \pm standard deviation ${ }^{\dagger}$ & $7 I \pm 7$ & $72 \pm 6$ & $71 \pm 8$ & 0.56 \\
\hline Range & $62-84$ & $67-83$ & $62-84$ & \\
\hline Baseline pain score $(\mathrm{TI})^{\dagger}$ (average daily pain - pain logbook) & $4.3 \pm 2.9$ & $6.3 \pm 3.1$ & $2.7 \pm 1.4$ & 0.10 \\
\hline \multicolumn{5}{|l|}{ Etiology of pain } \\
\hline Osteoarthritis & 4 & 3 & 1 & \\
\hline Sprained shoulder & I & & I & \\
\hline Chronic low back pain & 4 & 2 & 2 & \\
\hline Cervical injury & 1 & 1 & & \\
\hline Shoulder tendonitis & I & & I & \\
\hline Polymyalgia rheumatica & 1 & & I & \\
\hline Sciatica & I & & I & \\
\hline Unspecific leg pain & I & & I & \\
\hline Side of the most problematic pain (right/left) $)^{\ddagger}$ & $8 / 6$ & $4 / 2$ & $4 / 4$ & 0.41 \\
\hline \multicolumn{5}{|l|}{ Duration of chronic pain } \\
\hline Less than 2 years & 2 & I & I & \\
\hline Between 2 and 10 years & 3 & 1 & 2 & \\
\hline More than 10 years & 9 & 4 & 5 & \\
\hline Mean \pm standard deviation ${ }^{\dagger}$ & $20 \pm 18$ & $26 \pm 24$ & $|5 \pm I|$ & 0.48 \\
\hline Baseline ISI score ${ }^{\dagger}$ & $14.9 \pm 5.2$ & $15.0 \pm 6.4$ & $14.9 \pm 4.7$ & 0.39 \\
\hline Baseline sleep efficacy $(\mathrm{TI})^{\dagger}$ & $85.9 \pm 7.0$ & $86.4 \pm 7.5$ & $85.5 \pm 8.1$ & 0.83 \\
\hline Baseline quantity of nocturnal awakenings $(\mathrm{TI})^{\dagger}$ & $53.9 \pm 28.4$ & $50.2 \pm 32.3$ & $56.6 \pm 27.1$ & 0.90 \\
\hline \multicolumn{5}{|l|}{ Duration of sleep complaints } \\
\hline Less than 2 years & 2 & I & 1 & \\
\hline Between 2 and 10 years & 5 & I & 4 & \\
\hline More than 10 years & 7 & 4 & 3 & \\
\hline Mean \pm standard deviation ${ }^{\dagger}$ & $21 \pm 22$ & $28 \pm 25$ & $16 \pm 19$ & 0.44 \\
\hline \multicolumn{5}{|l|}{ Present medication } \\
\hline Pregabalin & 2 & I & I & \\
\hline Benzodiazepine & 4 & 3 & 1 & \\
\hline NSAID & 3 & I & 2 & \\
\hline Morphine & 1 & 1 & & \\
\hline SNRI & 2 & 2 & & \\
\hline Tricyclic antidepressant & 2 & 2 & & \\
\hline Levothyroxine & 4 & 2 & 2 & \\
\hline$\beta$-Blocker & 3 & 2 & 1 & \\
\hline ASA & 2 & I & I & \\
\hline PPI & 3 & 2 & I & \\
\hline Statin & 5 & 2 & 3 & \\
\hline ARB & 2 & I & I & \\
\hline Thiazide diuretic & I & & I & \\
\hline ACE inhibitor & I & & I & \\
\hline Triptan & I & & I & \\
\hline No medication & 2 & 1 & 1 & \\
\hline \multicolumn{5}{|l|}{ Other health problem } \\
\hline Myalgic encephalomyelitis & I & I & & \\
\hline Myocardial ischemia & 1 & 1 & & \\
\hline Hypercholesterolemia & 5 & 2 & 3 & \\
\hline Angina pectoris & I & I & & \\
\hline Hypertension & 4 & 2 & 2 & \\
\hline Hypothyroidism & 4 & 2 & 2 & \\
\hline No other health problem & 6 & 2 & 4 & \\
\hline
\end{tabular}

Notes: Some participants had more than I health problem and medication; consequently, the total number of health problems and medications does not correspond to the total number of participants in each group. *P-value of the comparison between active tDCS group and sham tDCS group. ${ }^{*}$ Fisher's exact test. ${ }^{+}$Mann-Whitney test. Data presented as mean \pm standard deviation or $n$.

Abbreviations: tDCS, transcranial direct current stimulation; F, female; M, male; ISI, Insomnia Severity Index; NSAID, nonsteroidal anti-inflammatory drug; SNRI, serotoninnorepinephrine reuptake inhibitor; ASA, acetylsalicylic acid; PPI, proton pump inhibitor; ARB, angiotensin II receptor blocker; ACE, angiotensin-converting enzyme; TI, 7-day baseline. 


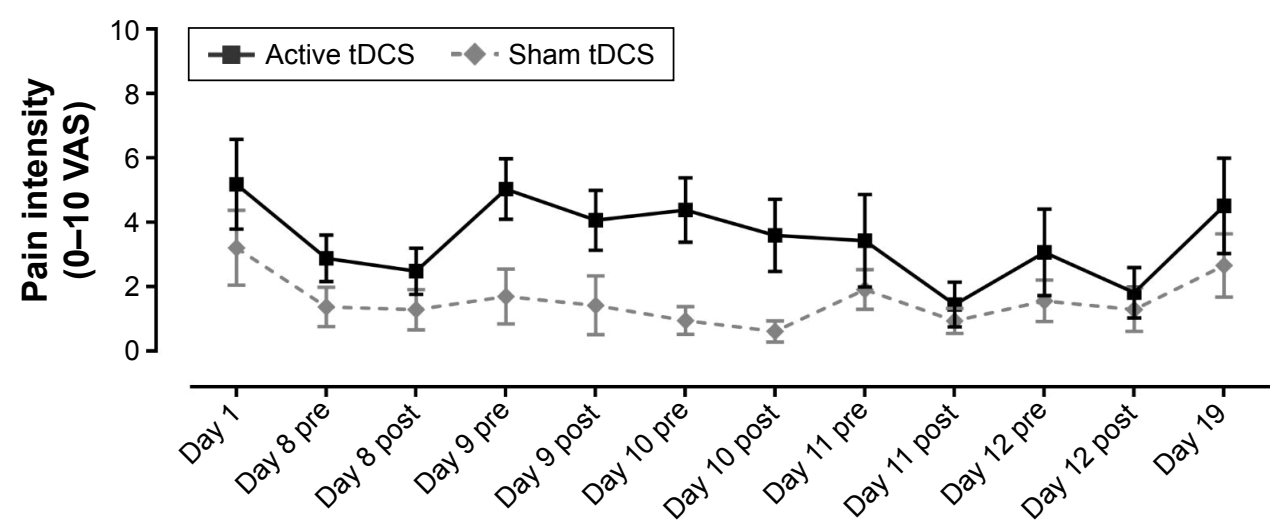

Figure 2 Pain intensity scores measured with VAS for active and sham tDCS groups. Pain scores were obtained once on day I and day I9, and before and after each tDCS session (day 8 to day 12). Each point represents group mean \pm standard error of mean.

Abbreviations: VAS, visual analog scale; tDCS, transcranial direct current stimulation.

Table 3 shows the results of pain questionnaires for each time point. Active tDCS generated a significant change in MPQ scores. Post hoc Wilcoxon signed-rank tests revealed that $\mathrm{tDCS}$ reduced MPQ scores at $\mathrm{T} 2$ and $\mathrm{T} 3$, when compared to baseline (T1) (all $P \leq 0.04$ ), suggesting that active tDCS can affect the qualitative aspects of pain. No changes over time were noted for physical functioning, as measured by the SF-BPI for both active and sham tDCS groups $(P \geq 0.07)$. There were no between-group differences, according to both questionnaires, for all time measures (all $P \geq 0.07$ ).

\section{Sleep outcomes}

The actigraphic measures generated several missing data, and the analyses of sleep efficiency and sleep-onset latency were

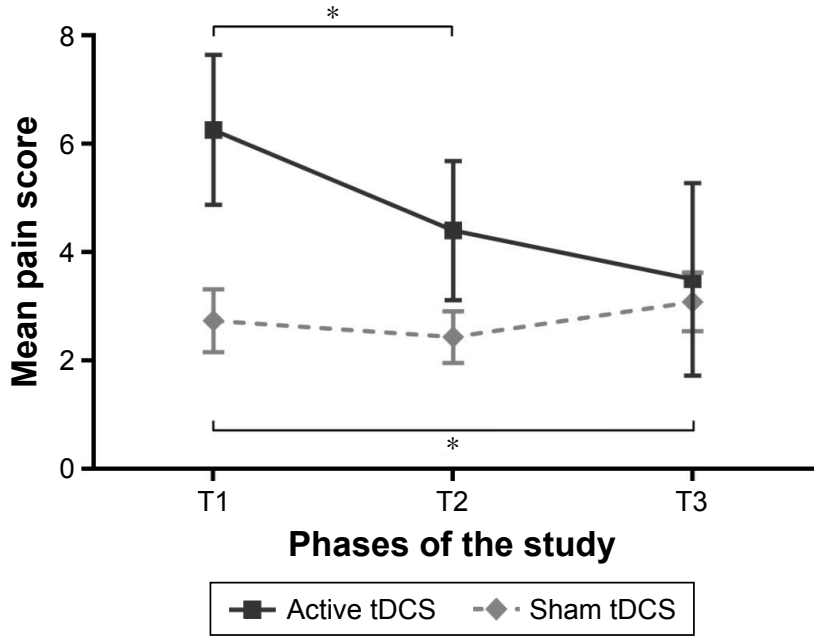

Figure 3 The average daily pain for sham and active treatment groups gathered using the pain logbook. TI represents the 7 days of baseline, T2 corresponds to the 5 days of tDCS treatments and T3 represents the 7 days of follow-up. Each point represents a group mean \pm standard error of mean. There was a significant difference between $\mathrm{TI}$ and $\mathrm{T} 2$ in the active tDCS group and between $\mathrm{TI}$ and $\mathrm{T} 3$ in sham tDCS group. *Statistically significant $(P<0.05)$.

Abbreviations: tDCS, transcranial direct current stimulation; TI, baseline; $\mathrm{T} 2$, tDCS treatments; T3, follow-up. done only for 4 participants ( 2 participants by group), which dangerously reduced the power of the statistical analysis. Still, visual inspection of the diagrams showed no trend toward a difference between the 2 participants who received active tDCS and the 2 participants who received sham tDCS. Intragroup inspection revealed that active tDCS seems to decrease sleep efficiency at T2 and T3 (approximately $-7 \%$ and $-1 \%$, respectively) (all $P \geq 0.20$ ), to increase sleep-onset latency at T2 (+12 minutes) and to decrease sleep latency at T3 ( -34 minutes) (all $P \geq 0.22$ ).

There were no missing data for the number of nocturnal awakenings evaluated with actigraphy and for the sleep diary measures and sleep questionnaires (PSQI and SF-BPI). There was no change in nocturnal awakenings at $\mathrm{T} 2$ and $\mathrm{T} 3$ when compared to $\mathrm{T} 1$ in the active tDCS group (approximately +4 and +5 awakenings, respectively), nor in the sham tDCS group

Table 2 Etiology of pain and daily average pain ratings of the 3 phases of the study

\begin{tabular}{|c|c|c|c|c|c|}
\hline \multirow[t]{2}{*}{ Participant } & \multirow[t]{2}{*}{ Group } & \multirow[t]{2}{*}{ Etiology of pain } & \multicolumn{3}{|c|}{ Pain score } \\
\hline & & & $\overline{T I}$ & T2 & T3 \\
\hline I & Active tDCS & Osteoarthritis & 8.3 & 6.3 & 4.9 \\
\hline 2 & & Cervical injury & 10.0 & 8.9 & 10 \\
\hline 3 & & Osteoarthritis & 8.6 & 2.1 & 1.2 \\
\hline 4 & & Osteoarthritis & 4.5 & 3.9 & 2.3 \\
\hline 5 & & Chronic low back pain & 3.4 & 3.0 & 1.2 \\
\hline 6 & & Chronic low back pain & 2.7 & 2.1 & 1.5 \\
\hline 7 & Sham tDCS & Sprained shoulder & 4.2 & 3.7 & 4.2 \\
\hline 8 & & Shoulder tendonitis & 1.3 & 1.3 & 1.6 \\
\hline 9 & & Osteoarthritis & 3.9 & 2.6 & 3.9 \\
\hline 10 & & Chronic low back pain & 1.0 & 0.8 & 1.4 \\
\hline 11 & & Polymyalgia rheumatica & 4.0 & 4.0 & 4.2 \\
\hline 12 & & Sciatica & 2.4 & 2.6 & 3.5 \\
\hline 13 & & Unspecific leg pain & 1.1 & 1.4 & 1.5 \\
\hline 14 & & Chronic low back pain & 4.0 & 3.2 & 4.3 \\
\hline
\end{tabular}

Abbreviations: tDCS, transcranial direct current stimulation; TI, baseline; $\mathrm{T} 2$, tDCS treatments; T3, follow-up. 


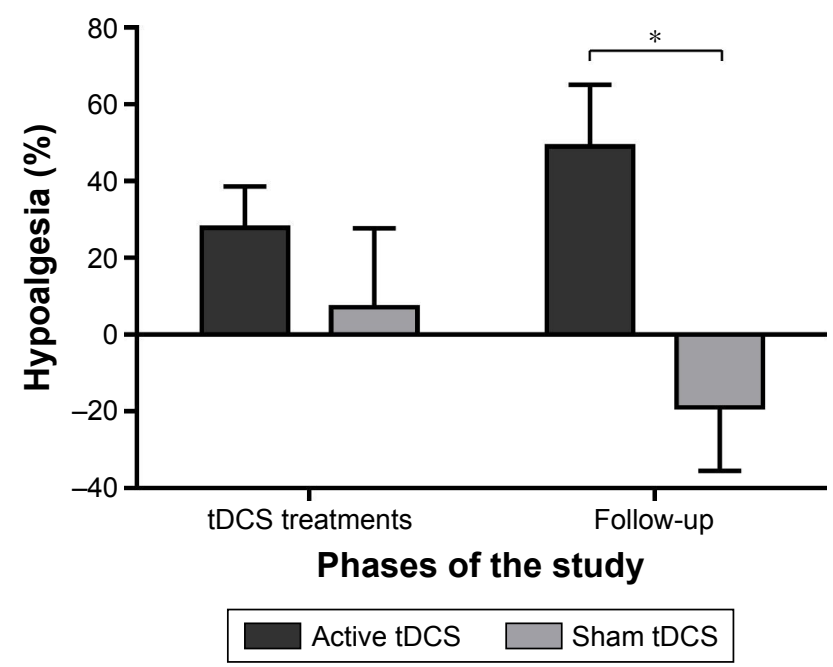

Figure 4 Percentages of hypoalgesia calculated with the average pain on the day measured using the pain logbook. The first 2 columns represent hypoalgesia during the week of tDCS treatments (comparing $\mathrm{T} 2$ to $\mathrm{TI}$ ), and the next 2 columns represent hypoalgesia during the 7 days of follow-up (comparing T3 to TI). Each column represents mean \pm standard error of mean. *Statistically significant $(P<0.05)$.

Abbreviations: tDCS, transcranial direct current stimulation; TI, baseline; T2, tDCS treatments; T3, follow-up.

(approximately -8 awakenings for both time measures) (all $P \geq 0.57$ ). Mann-Whitney tests showed that there were no between-group differences in nocturnal awakenings for all time measures (all $P \geq 0.69$ ). The data collected with the sleep diaries revealed that patients observed no difference in their sleep during or after the active and sham tDCS treatments. Table 3 shows that there was no change in sleep questionnaires scores during and after tDCS when compared to baseline, for both the active and sham tDCS group (all $P \geq 0.12$ ). MannWhitney tests also showed that there were no between-group differences for all time measures (all $P \geq 0.22$ ).

\section{Discussion}

The first objective of the present study was to provide information regarding the feasibility of conducting a randomized, double-blind, sham-controlled trial considering the efficacy of tDCS to reduce pain and improve sleep in older adults suffering from chronic musculoskeletal pain. Although no major obstacles prevented the completion of the study, some important recommendations can be made. First, using a stratified randomization strategy for key factors (eg, baseline pain levels) appears to be of primary importance. ${ }^{49,50}$ This is particularly true for small-sample-size studies, as conventional simple randomization methods can generate imbalances in baseline characteristics among groups. ${ }^{49,50}$ In the present study, despite randomization, the group that received active tDCS treatments had higher levels of baseline pain, compared to the group that received sham tDCS. Even if this difference was not statistically significant, baseline differences in core outcome measures are always problematic. In the present case, it remains possible that the pattern of results observed (ie, greater effect of active tDCS than sham tDCS) is partly attributable to the fact that it is easier to provide pain relief when initial pain is high ${ }^{48}$ Despite the fact that increasing the number of participants could help attenuate baseline group differences, we suggest that future studies should randomize patients according to their initial pain. Second, although pain measures and questionnaires were correctly understood by the participants, these measures often required several explanations before participants could fully grasp the concept evaluated. Even though the pain scales and questionnaires used in this study (eg, VAS, MPQ, Brief Pain Inventory) are

Table 3 Pain and sleep questionnaires

\begin{tabular}{|c|c|c|c|c|c|}
\hline \multirow[t]{2}{*}{ Questionnaire } & \multirow[t]{2}{*}{ tDCS group } & \multicolumn{3}{|l|}{ Scores } & \multirow[t]{2}{*}{$\boldsymbol{P}_{\text {-value }}^{\dagger}$} \\
\hline & & Baseline (day I) & Post-tDCS (day I 2) & Follow-up (day I9) & \\
\hline \multirow[t]{3}{*}{ MPQ } & Active & $32.2 \pm 13.7$ & $16.2 \pm 8.1$ & $21.5 \pm 17.6$ & $0.02 *$ \\
\hline & Sham & $23.8 \pm 16.3$ & $24.4 \pm \mid 4.1$ & $22.8 \pm 7.8$ & 0.80 \\
\hline & $P$-value $\ddagger$ & 0.25 & 0.22 & 0.80 & \\
\hline \multirow[t]{3}{*}{ SF-BPI } & Active & $29.0 \pm 11.0$ & $12.2 \pm 10.2$ & $17.6 \pm 17.3$ & 0.07 \\
\hline & Sham & $17.1 \pm 10.3$ & II. $.0 \pm 8.7$ & 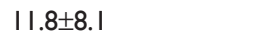 & 0.17 \\
\hline & $P$-value $\ddagger$ & 0.07 & 0.80 & 0.56 & \\
\hline \multirow[t]{3}{*}{ SF-BPI (pain interference with sleep) } & Active & $6.5 \pm 1.9$ & $3.3 \pm 3.4$ & $5.2 \pm 5.0$ & 0.12 \\
\hline & Sham & $4.8 \pm 2.5$ & $3.0 \pm 2.4$ & $3.8 \pm 2.8$ & 0.24 \\
\hline & $P$-value ${ }^{\ddagger}$ & 0.22 & 0.90 & 0.77 & \\
\hline \multirow[t]{3}{*}{ PSQI } & Active & $12.0 \pm 2.8$ & $10.2 \pm 3.9$ & $10.3 \pm 6.6$ & 0.57 \\
\hline & Sham & $9.8 \pm 3.9$ & $9.9 \pm 2.4$ & $8.0 \pm 2.4$ & 0.12 \\
\hline & $P$-value ${ }^{\ddagger}$ & 0.24 & 0.84 & 0.40 & \\
\hline
\end{tabular}

Notes: Data are presented as mean value \pm standard deviation and $95 \%$ confidence interval. ${ }^{\dagger}$ Friedman tests were performed to detect intragroup differences. *Statistically significant. ¥Mann-Whitney tests were performed to detect between-group differences.

Abbreviations: tDCS, transcranial direct current stimulation; MPQ, McGill Pain Questionnaire; SF-BPI, Short Form of the Brief Pain Inventory; PSQI, Pittsburgh Sleep Quality Index. 
designed to be self-administered, these observations indicate that the presence of a member of the research team is essential to ensure valid pain measures in studies conducted in elderly individuals. Of importance, the results show that the use of NRS (included in the pain logbooks) is appropriate to evaluate changes in the daily pain of elderly chronic pain patients. NRS appears to be a simpler tool to understand than VAS, an observation that is coherent with that of Dworkin et $\mathrm{al}^{33}$ and Herr. ${ }^{51}$

Blinding of the participants and of the evaluators is always a major concern of randomized controlled studies. In a commendable study published recently by O'Connell et al, the authors suggest that the use of a sham tDCS treatment, applied at an intensity of $2 \mathrm{~mA}$, is hardly attainable. ${ }^{52}$ Indeed, based on the sensation of the stimulation, $65 \%$ of the participants correctly judged the stimulation condition (active or sham), while evaluators noticed skin changes (redness) under the reference electrode more often following active tDCS than sham tDCS. ${ }^{52}$ In our case, none of the participants, nor the evaluator, were able to distinguish between the 2 types of stimulation (sham or active tDCS). ${ }^{52}$ Contrary to O'Connell et al, the participants included in this study were elderly individuals. Perhaps the age-related changes observed in tegumentary and sensory functions (eg, decreased sensation and skin thickness) could explain these discrepancies. ${ }^{53-55}$ Indeed, all the participants (including those who received sham tDCS, and therefore, only 60 seconds in total of real stimulation) presented some redness under the reference electrode.

Participants wore the actigraph without problems during the 19 days of the study. Although some studies consider that actigraphy is a valid method to assess sleep, it is important to mention that in our study, the use of actigraphy led to many missing data. ${ }^{56,57}$ The analyses revealed that many participants tended to be immobile in bed, even when awake, thus leading to several incorrect or missing data concerning sleep-onset latency (a measure that is also used to calculate sleep efficiency). Consequently, measuring sleep efficiency with actigraphy is probably not the best strategy in older populations. Although costlier and more time consuming, future studies should consider using polysomnography instead of actigraphy. ${ }^{58}$ However, the absence of changes noted in the subjective measures of sleep (sleep diaries and questionnaires) sheds some doubt on the potential utility of tDCS to decrease sleep problems in elderly individuals and somewhat diminishes the interest for the implementation of a polysomnography study in this population. As for pain questionnaires, many participants needed help to complete the sleep questionnaires, highlighting once again the key role of the research team.

\section{Effect of tDCS on pain intensity}

Among the active tDCS group, there was a significant reduction in daily average pain during tDCS treatments (T2), when compared to baseline (T1). Of importance, the reduction in pain observed at $\mathrm{T} 2$ was clinically significant for patients suffering from chronic musculoskeletal pain. ${ }^{48}$ Moreover, despite the fact that the reduction of the average daily pain in the follow-up (T3) was not statistically significant when compared with $\mathrm{T} 1$, it is important to note that the pain reduction provided by the active tDCS between T1 and $\mathrm{T} 3$ is almost 3 points on the NRS. Thus, this reduction in the average daily pain is clinically significant and associated with the concept of a "much better" improvement in pain. ${ }^{48,59}$ The large variability in tDCS-induced pain reductions observed at $\mathrm{T} 3$ in the active tDCS group can probably explain why this important difference did not reach statistical significance. Concerning the sham tDCS group, there was an increase in the average daily pain at T3 when compared to T1. Although these differences are statistically significant, they are not clinically significant.

Many of the previous studies considering the analgesic efficacy of tDCS were conducted with young or ageheterogeneous populations, and on patients suffering from neuropathic pain. In one of the first studies published on the effect of tDCS on pain, Fregni et $\mathrm{al}^{21}$ evaluated the analgesic efficacy of tDCS in a group of 17 subjects with spinal cord injuries (mean age: $35.7 \pm 13.3$ years). The authors reported that tDCS, applied for 20 minutes at $2 \mathrm{~mA}$ for 5 consecutive days, reduced pain in this patient population, suggesting that this stimulation protocol is effective in adults suffering from neuropathic pain, a conclusion that was substantiated by many other studies..$^{23,42}$ These results contrast with those of Wrigley et al who observed no effect of tDCS on the pain in patients suffering from neuropathic pain conditions. ${ }^{60}$ Very few studies looked into the analgesic efficacy of tDCS on chronic musculoskeletal pain. Antal et al have assessed the analgesic quality of tDCS in patients suffering from chronic musculoskeletal or neuropathic pain (age range: $41-70$ years). ${ }^{22}$ They reported that anodal tDCS produced a $30 \%$ reduction in pain after the fifth and last session of stimulation. Interestingly, this decrease in pain after 5 anodal sessions of tDCS was like that of the present study (average reduction of $28 \%$ when comparing T2 to T1). For their part, Schabrun et al observed a decrease in pain for almost $60 \%$ of patients with chronic low back pain (CLBP) (mean age: $30 \pm 2$ years). ${ }^{61}$ However, 
the age difference between the population in Schabrun et al's study and the current study makes any comparison somewhat hazardous. A recent study published by Concerto et al assessed the analgesic efficiency of tDCS on 10 elderly patients with plantar fasciitis (mean age: $68.8 \pm 3.3$ years) ${ }^{62}$ This study showed a decrease in pain that was relatively identical to that of the current study $(36.9 \%$ vs $28 \%$ during the 5 days of treatment and $42.4 \%$ vs $49 \%$ on the week after the treatment). To our knowledge, Concerto et al's pilot study is the only other study that looked at the analgesic effect of tDCS in elderly individuals. Although interesting, it should be noted that the results of Concerto et al were obtained using an open-label design, with no placebo condition. This can be problematic given the important placebo effect noted in studies looking into the analgesic effect of brain stimulation techniques. ${ }^{63}$

The long-term effect of tDCS has been measured in several studies. In one of their studies, Fregni et al reported that the pain improvement induced by 5 sessions of tDCS in women with fibromyalgia was still apparent 3 weeks after the stimulation sessions. ${ }^{64}$ Similarly, Valle et al demonstrated that 10 sessions of tDCS could reduce pain in women suffering from fibromyalgia (mean age: 54.8 9 9.6 years) for a period of 60 days, whereas Auvichayapat et al reported that 20 sessions of tDCS produced a decrease in pain for a period of 12 weeks in patients suffering from migraines (mean age: $28.6 \pm 6.8$ years) ${ }^{65,66}$ Increasing the number of tDCS sessions could produce cumulative and more long-lasting effects. ${ }^{67}$ In contrast, O'Connell et al showed that $\mathrm{tDCS}$ did not produce a significant effect on the pain in patients with CLBP (mean age: $45 \pm 10$ years), regardless of the number of sessions. ${ }^{68}$

\section{Effect of tDCS on sleep}

To our knowledge, only 1 study specifically focused on the effect of tDCS on sleep structure in patients suffering from chronic pain. In this study, Roizenblatt et al aimed to determine if the application of tDCS in women suffering from fibromyalgia could decrease pain, as well as improve sleep quality. ${ }^{20}$ Using polysomnography, the authors observed that 5 sessions of anodal tDCS stimulation applied over the primary motor cortex improved sleep parameters and architecture in patients suffering from fibromyalgia. In the present study, no benefits were observed on sleep variables following tDCS applications. It is important to note that, although the populations examined in this study and in the study of Roizenblatt et al both suffered from chronic pain, the type of pain, as well as the sex and the age of participants, was quite different. ${ }^{20}$ Past research has shown that sleep mechanisms are different in elderly individuals when compared to young adults (eg, sleep-wake cycle, more frequent and longer periods of awakening, wake-up time in the morning, sleep satisfaction, percentage of slow-wave sleep and rapid eye movement sleep). ${ }^{11,14,69-72}$ These differences may explain why tDCS did not have an impact on the elderly, whereas it was effective in younger adults. Furthermore, contrary to fibromyalgia (in which sleep disorder is generally recognized as a primary symptom ${ }^{73}$ ), the sleep disorders experienced by the participants of this study were secondary to pain problems. Perhaps, a longer follow-up would have allowed us to detect sleep changes in our participants.

Although they did not assess the effect of tDCS on sleep structure, other authors have focused on the effect that tDCS has on sleep. In one of these studies, Borckardt et al measured the analgesic effect of tDCS in patients who had undergone endoscopic retrograde cholangiopancreatography (ERCP). ${ }^{26}$ They found that the patients who received real tDCS (10 women, mean age: $37.8 \pm 10.8$ years) reported less pain interference with sleep (according to 1 question of the BPI) than those who received sham tDCS. In the present study, no change was noted following tDCS for the same question from the BPI. It is however important to note that the results of Borckardt et al's study are the result of the analyses of only 24 hours post-ERCP.

\section{Conclusion}

The present study provides preliminary evidence on the efficacy of tDCS to reduce pain and improve sleep in older adults, as well as guidelines for the implementation of future studies. tDCS appears to be a promising therapeutic avenue for older individuals suffering from chronic musculoskeletal pain. Future studies evaluating the effect of tDCS on sleep should consider the shortcomings of actigraphic measures and should strongly consider using polysomnography.

\section{Acknowledgments}

The authors would like to thank Ms Marie-Claude Girard, Mr Antoine Guillerand and Ms Isabelle Viens for their help with data collection, as well as Mr Yannick TousignantLaflamme and Ms Anaïs Lacasse for their thoughtful comments on the manuscript. They also thank all the subjects who participated in this project. Part of this work served as an M.Sc. degree fulfillment by Marie-Philippe Harvey. Preliminary results of this research were presented as a poster at the International Neuromodulation Society 12th World Congress Neuromodulation: Medicine Evolving Through Technology in June 2015, and the abstract was published 
online at www.onlinelibrary.wiley.com/doi/10.1111/ ner.12333/abstract. G Léonard is supported by the Fonds de Recherche en Santé (FRQ-S, Montréal, QC, Canada). This project was partially supported by the Neuroscience Centre of Excellence of the Université de Sherbrooke (CeNUS, Sherbrooke, QC, Canada) and an internal start-up fund from the Research Centre on Aging (Initiatives stratégiques du Centre de recherche sur le vieillissement, Sherbrooke, QC, Canada).

\section{Disclosure}

The authors report no conflicts of interest in this work.

\section{References}

1. Manocchia M, Keller S, Ware JE. Sleep problems, health-related quality of life, work functioning and health care utilization among the chronically ill. Oual Life Res. 2001;10(4):331-345.

2. Marchand S. Le phénomène de la douleur [The Phenomenon of Pain]. Montreal: Les Éditions de la Chenelière Inc; 2009. French.

3. Cho CH, Jung SW, Park JY, Song KS, Yu KI. Is shoulder pain for three months or longer correlated with depression, anxiety, and sleep disturbance? J Shoulder Elbow Surg. 2013;22(2):222-228.

4. Fishbain DA, Cole B, Lewis JE, Gao J. What is the evidence for chronic pain being etiologically associated with the DSM-IV category of sleep disorder due to a general medical condition? A structured evidencebased review. Pain Med. 2010;11(2):158-179.

5. Onen SH, Onen F, Courpron P, Dubray C. How pain and analgesics disturb sleep. Clin J Pain. 2005;21(5):422-431.

6. Purushothaman B, Singh A, Lingutla K, Bhatia C, Pollock R, Krishna M. Prevalence of insomnia in patients with chronic back pain. $J$ Orthop Surg (Hong Kong). 2013;21(1):68-70.

7. Schuh-Hofer S, Wodarski R, Pfau DB, et al. One night of total sleep deprivation promotes a state of generalized hyperalgesia - a surrogate pain model to study the relationship of insomnia and pain. Pain. 2013; 154(9):1613-1621.

8. Stiefel F, Stagno D. Management of insomnia in patients with chronic pain conditions. CNS Drugs. 2004;18(5):285-296.

9. Morin CM, LeBlanc M, Daley M, Gregoire JP, Merette C. Epidemiology of insomnia: prevalence, self-help treatments, consultations, and determinants of help-seeking behaviors. Sleep Med. 2006;7(2):123-130.

10. Smith MT, Haythornthwaite JA. How do sleep disturbance and chronic pain inter-relate? Insights from the longitudinal and cognitive-behavioral clinical trials literature. Sleep Med. 2004;8(2):119-132.

11. Crowley K. Sleep and sleep disorders in older adults. Neuropsychology Rev. 2011;21(1):41-53.

12. Gibson SJ, Lussier D. Prevalence and relevance of pain in older persons. Pain Med. 2012;13(Suppl 2):S23-S26.

13. Helme RD, Gibson SJ. The epidemiology of pain in elderly people. Clin Geriatr Med. 2001;17(3):417-431.

14. Rissling MaA-ISUoCaSD. Sleep in aging. In: Handbook of the Neuroscience of Aging. 2009:373-379.

15. Beaulieu P, Lussier D, Porreca F, Dickenson AH. Pharmacology of Pain. Seattle: IASP Press; 2010.

16. Marchand S. Pharmacologie de la douleur [Pharmacology of Pain]. 1st ed. Beaulieu P, editor. Montreal: Presses de l'Université de Montréal; 2005. French.

17. Roehrs T, Roth T. Insomnia pharmacotherapy. Neurotherapeutics. 2012;9(4):728-738.

18. Benninger D, Kuntzer T. Treatment of chronic pain: transcranial stimulation of the motor cortex? Rev Med Suisse. 2012;8(339):935-936.
19. Ballentine NH. Polypharmacy in the elderly: maximizing benefit, minimizing harm. Crit Care Nurs Q. 2008;31(1):40-45.

20. Roizenblatt S, Fregni F, Gimenez R, et al. Site-specific effects of transcranial direct current stimulation on sleep and pain in fibromyalgia: a randomized, sham-controlled study. Pain Pract. 2007;7(4): 297-306.

21. Fregni F, Boggio PS, Lima MC, et al. A sham-controlled, phase II trial of transcranial direct current stimulation for the treatment of central pain in traumatic spinal cord injury. Pain. 2006;122(1-2):197-209.

22. Antal A, Terney D, Kühnl S, Paulus W. Anodal transcranial direct current stimulation of the motor cortex ameliorates chronic pain and reduces short intracortical inhibition. J Pain Symptom Manage. 2010;39(5): 890-903.

23. Kim YJ, Ku J, Kim HJ, et al. Randomized, sham controlled trial of transcranial direct current stimulation for painful diabetic polyneuropathy. Ann Rehabil Med. 2013;37(6):766-776.

24. Yoon EJ, Kim YK, Kim HR, Kim SE, Lee Y, Shin HI. Transcranial direct current stimulation to lessen neuropathic pain after spinal cord injury: a mechanistic pet study. Neurorehabil Neural Repair. 2014;28(3): 250-259.

25. Borckardt JJ, Reeves ST, Robinson SM, et al. Transcranial direct current stimulation (tDCS) reduces postsurgical opioid consumption in total knee arthroplasty (TKA). Clin J Pain. 2013;29(11):925-928.

26. Borckardt JJ, Romagnuolo J, Reeves ST, et al. Feasibility, safety, and effectiveness of transcranial direct current stimulation for decreasing post-ERCP pain: a randomized, sham-controlled, pilot study. Gastrointest Endosc. 2011;73(6):1158-1164.

27. De Vis JB, Hendrikse J, Bhogal A, Adams A, Kappelle LJ, Petersen ET. Age-related changes in brain hemodynamics; a calibrated MRI study. Hum Brain Mapp. 2015;36(10):3973-3987.

28. Sowell ER, Peterson BS, Thompson PM, Welcome SE, Henkenius AL, Toga AW. Mapping cortical change across the human life span. Nat Neurosci. 2003;6(3):309-315.

29. Crivello F, Tzourio-Mazoyer N, Tzourio C, Mazoyer B. Longitudinal assessment of global and regional rate of grey matter atrophy in 1,172 healthy older adults: modulation by sex and age. PLoS One. 2014; 9(12):e114478.

30. Clark BC, Taylor JL. Age-related changes in motor cortical properties and voluntary activation of skeletal muscle. Curr Aging Sci. 2011;4(3): 192-199.

31. Morin CM, Belleville G, Belanger L, Ivers H. The insomnia severity index: psychometric indicators to detect insomnia cases and evaluate treatment response. Sleep. 2011;34(5):601-608.

32. Price DD, McGrath PA, Rafii A, Buckingham B. The validation of visual analogue scales as ratio scale measures for chronic and experimental pain. Pain. 1983;17(1):45-56.

33. Dworkin RH, Turk DC, Farrar JT, et al; IMMPACT. Core outcome measures for chronic pain clinical trials: IMMPACT recommendations. Pain. 2005;113(1-2):9-19.

34. Wood BM, Nicholas MK, Blyth F, Asghari A, Gibson S. Assessing pain in older people with persistent pain: the NRS is valid but only provides part of the picture. J Pain. 2010;11(2):1259-1266.

35. Melzack R. The McGill pain questionnaire: major properties and scoring methods. Pain. 1975;1(3):277-299.

36. Mendoza T, Mayne T, Rublee D, Cleeland C. Reliability and validity of a modified brief pain inventory short form in patients with osteoarthritis. Eur J Pain. 2006;10(4):353-361.

37. Poundja J, Fikretoglu D, Guay S, Brunet A. Validation of the French version of the brief pain inventory in Canadian veterans suffering from traumatic stress. J Pain Symptom Manage. 2007;33(6):720-726.

38. Veilleux SSD, Bohuon A. Le défi de la douleur [The Challenge of Pain]. 3rd ed. Melzack R, Wall PD, editors. Edisem; Saint-Hyacinthe, QC, Canada. 1989. French.

39. Marino M, Li Y, Rueschman MN, et al. Measuring sleep: accuracy, sensitivity, and specificity of wrist actigraphy compared to polysomnography. Sleep. 2013;36(11):1747-1755. 
40. Sadeh A, Hauri PJ, Kripke DF, Lavie P. The role of actigraphy in the evaluation of sleep disorders. Sleep. 1995;18(4):288-302.

41. Mollayeva T, Thurairajah P, Burton K, Mollayeva S, Shapiro CM, Colantonio A. The Pittsburgh sleep quality index as a screening tool for sleep dysfunction in clinical and non-clinical samples: a systematic review and meta-analysis. Sleep Med Rev. 2016;25:52-73.

42. Mori F, Codeca C, Kusayanagi H, et al. Effects of anodal transcranial direct current stimulation on chronic neuropathic pain in patients with multiple sclerosis. J Pain. 2010;11(5):436-442.

43. Nitsche MA, Paulus W. Excitability changes induced in the human motor cortex by weak transcranial direct current stimulation. J Physiol. 2000;527(Pt 3):633-639.

44. Gandiga PC, Hummel FC, Cohen LG. Transcranial DC stimulation (tDCS): a tool for double-blind sham-controlled clinical studies in brain stimulation. Clin Neurophysiol. 2006;117(4):845-850.

45. Kessler SK, Turkeltaub PE, Benson JG, Hamilton RH. Differences in the experience of active and sham transcranial direct current stimulation. Brain Stimul. 2012;5(2):155-162.

46. Palm U, Reisinger E, Keeser D, et al. Evaluation of sham transcranial direct current stimulation for randomized, placebo-controlled clinical trials. Brain Stimul. 2013;6(4):690-695.

47. Farrar JT, Portenoy RK, Berlin JA, Kinman JL, Strom BL. Defining the clinically important difference in pain outcome measures. Pain. 2000; 88(3):287-294.

48. Salaffi F, Stancati A, Silvestri CA, Ciapetti A, Grassi W. Minimal clinically important changes in chronic musculoskeletal pain intensity measured on a numerical rating scale. Eur J Pain. 2004;8(4):283-291.

49. Altman DG, Bland JM. How to randomise. BMJ. 1999;319(7211): 703-704.

50. Kang M, Ragan BG, Park JH. Issues in outcomes research: an overview of randomization techniques for clinical trials. $J$ Athl Train. 2008; 43(2):215-221.

51. Herr K. Pain assessment strategies in older patients. J Pain. 2011; 12(3 Suppl 1):S3-S13.

52. O'Connell NE, Cossar J, Marston L, et al. Rethinking clinical trials of transcranial direct current stimulation: participant and assessor blinding is inadequate at intensities of $2 \mathrm{~mA}$. PLoS One. 2012;7(10):e47514.

53. O’Sullivan SB, Schmitz TJ, Behrman AL. Physical Rehabilitation. Philadelphia: F.A. Davis; 2007.

54. Bowden JL, McNulty PA. Age-related changes in cutaneous sensation in the healthy human hand. Age (Dordr). 2013;35(4):1077-1089.

55. Firooz A, Rajabi-Estarabadi A, Zartab H, Pazhohi N, Fanian F, Janani L. The influence of gender and age on the thickness and echo-density of skin. Skin Res Technol. 2017;23(1):13-20.

56. Blackwell T, Redline S, Ancoli-Israel S, et al. Comparison of sleep parameters from actigraphy and polysomnography in older women: the SOF study. Sleep. 2008;31(2):283-291.

57. Martin JL, Hakim AD. Wrist actigraphy. Chest. 2011;139(6):1514-1527.
58. Knauert MP, Yaggi HK, Redeker NS, Murphy TE, Araujo KL, Pisani MA. Feasibility study of unattended polysomnography in medical intensive care unit patients. Heart Lung. 2014;43(5):445-452.

59. Haefeli M, Elfering A. Pain assessment. Eur Spine J. 2006;15(Suppl 1): S17-S24.

60. Wrigley PJ, Gustin SM, McIndoe LN, Chakiath RJ, Henderson LA, Siddall PJ. Longstanding neuropathic pain after spinal cord injury is refractory to transcranial direct current stimulation: a randomized controlled trial. Pain. 2013;154(10):2178-2184.

61. Schabrun SM, Jones E, Elgueta Cancino EL, Hodges PW. Targeting chronic recurrent low back pain from the top-down and the bottom-up: a combined transcranial direct current stimulation and peripheral electrical stimulation intervention. Brain Stimul. 2014;7(3):451-459.

62. Concerto C, Al Sawah M, Chusid E, et al. Anodal transcranial direct current stimulation for chronic pain in the elderly: a pilot study. Aging Clin Exp Res. 2016;28(2):231-237.

63. de Campos GC. Placebo effect in osteoarthritis: why not use it to our advantage? World J Orthop. 2015;6(5):416-420.

64. Fregni F, Gimenes R, Valle AC, et al. A randomized, sham-controlled, proof of principle study of transcranial direct current stimulation for the treatment of pain in fibromyalgia. Arthritis Rheum. 2006;54(12): 3988-3998.

65. Valle A, Roizenblatt S, Botte S, et al. Efficacy of anodal transcranial direct current stimulation (tDCS) for the treatment of fibromyalgia: results of a randomized, sham-controlled longitudinal clinical trial. J Pain Manag. 2009;2(3):353-361.

66. Auvichayapat $P$, Janyacharoen T, Rotenberg A, et al. Migraine prophylaxis by anodal transcranial direct current stimulation, a randomized, placebo-controlled trial. J Med Assoc Thai. 2012;95(8):1003-1012.

67. Charvet LE, Kasschau M, Datta A, et al. Remotely-supervised transcranial direct current stimulation (tDCS) for clinical trials: guidelines for technology and protocols. Front Syst Neurosci. 2015;9:26.

68. O’Connell NE, Cossar J, Marston L, et al. Transcranial direct current stimulation of the motor cortex in the treatment of chronic nonspecific low back pain: a randomized, double-blind exploratory study. Clin $J$ Pain. 2013;29(1):26-34.

69. Lorrain D, Boivin D. Précis pratique de gériatrie [Precis Practice of Geriastrics]. 3rd ed. Arcand M, Hébert R, editors. Acton Vale: Edisem; 2007. French.

70. Landolt HP, Dijk DJ, Achermann P, Borbély AA. Effect of age on the sleep EEG: slow-wave activity and spindle frequency activity in young and middle-aged men. Brain Res. 1996;738(2):205-212.

71. Landolt HP, Borbély AA. Age-dependent changes in sleep EEG topography. Clin Neurophysiol. 2001;112(2):369-377.

72. Millman RP. Sleep and aging. Med Health R I. 2012;95(3):88-90.

73. Chakrabarty S, Zoorob R. Fibromyalgia. Am Fam Physician. 2007; 76(2):247-254.
Clinical Interventions in Aging

\section{Publish your work in this journal}

Clinical Interventions in Aging is an international, peer-reviewed journal focusing on evidence-based reports on the value or lack thereof of treatments intended to prevent or delay the onset of maladaptive correlates of aging in human beings. This journal is indexed on PubMed Central, MedLine,

\section{Dovepress}

CAS, Scopus and the Elsevier Bibliographic databases. The manuscript management system is completely online and includes a very quick and fair peer-review system, which is all easy to use. Visit http://www.dovepress. com/testimonials.php to read real quotes from published authors. 